\title{
Reviewers for Kew Bulletin Volume 72
}

We are grateful to the following people who reviewed articles for Kew Bulletin during the years 2016 - 2017.
P. Acevedo
D. Albach
F. Almeda
F. Barrie
M. Bunger
M. T. Buril
T. Daniel
Y.-F. Deng
I. Denholm
D. Desjardin
E. Fischer
B. Ford
I. Friis
H. Huaylla
M. Hughes
M. Kato
M. Lavin
G. Levin
J. Lovo
O. G. Martinez
L. Martinez-Suz
A. McDonald
J. Meirelles

D. Middleton

R. Mill

T. M. de Moura

M. Newman

L. Nusbaumer

J. Parnell

P. Pornpongrungrueng

C. Randle

L. Raz

Z. Rogers

M.-S. Samain

Sebsebe Demissew

G. Staples

S. Suddee

N. Tanaka

G. Tucker

H. van der Werff

L. Vasconcelos

R. Watling

M. Watson

J. R. I Wood

C. Zickel

F. Zuloaga 